\title{
Shock hemorrágico como presentación inicial de divertículo de Meckel
}

\author{
Camila Haro, Alexandra Duffau, Nicolás Muniz, Sofía Mansilla, \\ Manuel Sanguinetti, Marcelo Viola \\ Departamento de Cirugía Médica Uruguaya (MUCAM). Montevideo, Uruguay.
}

\begin{abstract}
RESUMEN
Introducción: El divertículo de Meckel es una anomalía congénita frecuente que suele ser asintomática, pero puede dar lugar a complicaciones graves. La presencia de mucosa heterotópica gástrica o pancreática en el divertículo favorece la ulceración del mismo, que puede causar una hemorragia digestiva.

Nuestro objetivo es mostrar la presentación de un divertículo de Meckel con una hemorragia digestiva baja con shock
\end{abstract} hemorrágico.

Caso clínico: Paciente de 34 años, que se presenta con dolor abdominal y enterorragia, asociados a anemia con $\mathrm{Hb}$ 7,9. En la evolución se instala una hemorragia masiva con shock hipovolémico, que responde a medidas de reanimación. Se solicita una arteriografía de vasos mesentéricos, que visualiza arteria irregular y tortuosa dependiente de la mesentérica superior que se emboliza. A las $48 \mathrm{~h}$ el paciente reitera el dolor abdominal y se realiza una laparoscopía que evidencia un divertículo de Meckel con signos de isquemia. Se reseca el mismo en cuña, con buena evolución posterior.

Discusión: La hemorragia digestiva es un desafío diagnóstico y terapéutico. De presentarse el paciente con inestabilidad hemodinámica el estudio de elección es la angiotomografía y eventual embolización del vaso sangrante. El divertículo de Meckel es una causa rara de hemorragia digestiva en el adulto. La angioembolización puede ser utilizada como contemporizadora a la cirugía, permitiendo estabilizar al paciente del punto de vista hemodinámico.

Conclusiones: Se presentó el caso clínico de un paciente con un shock hemorrágico secundario al sangrado de un divertículo de Meckel. El mismo fue tratado mediante angioembolización y posterior cirugía por isquemia local.

Palabras claves: Divertículo de Meckel; Hemorragia digestiva; Angioembolización

\begin{abstract}
Meckel's diverticulum is a common congenital anomaly, usually asymptomatic, although it can lead to serious complications. The presence of heterotopic gastric or pancreatic mucosa in the diverticulum favors its ulceration and can cause gastrointestinal bleeding. We present a 34-year-old man who consulted in the emergency room for abdominal pain, enterorrhagia, and anemia (Hb $7.9 \mathrm{~g} / \mathrm{dl}$ ). Subsequently, he developed a massive hemorrhage with hypovolemic shock. Arteriography showed an irregular and tortuous artery dependent on the superior mesenteric artery that was treated with embolization. Laparoscopy was performed at $48 \mathrm{~h}$ due to recurrence of abdominal pain, which revealed a Meckel's diverticulum with signs of ischemia, which was excised with good outcome.

Gastrointestinal bleeding originating from a Meckel's diverticulum is rare in adults. If the patient presents hemodynamic instability, the study of choice is CT angiography. Angioembolization can be used as a bridge to surgery, allowing the patient to hemodynamically stabilize.
\end{abstract}

Keywords: Meckel's Diverticulum; Gastrointestinal Bleeding; Angioembolization

\section{INTRODUCCIÓN}

El divertículo de Meckel es una anomalía congénita frecuente, que resulta de la atrofia incompleta del ducto vitelino durante el desarrollo embrionario. ${ }^{1}$ Se trata de un receso de fondo ciego localizado en el borde antimesentérico del íleon, a nivel de la desembocadura de la rama terminal de la arteria mesentérica superior. ${ }^{2}$

La mayor parte de estos divertículos no causa síntomas ni complicaciones durante la vida del individuo, pero pueden ser fuente de complicaciones graves. ${ }^{1,2}$

En ocasiones existe un desarrollo de mucosa ectópica en este divertículo, generalmente gástrica y, en menor medida, pancreática, duodenal, colónica, endometrial o hepatobiliar. $^{2}$

\footnotetext{
Los autores declaran ausencia de conflictos de interés.

Camila Haro

harob.camila@gmail.com

Recibido: octubre de 2020. Aceptado: noviembre de 2020.
}

En aquellos divertículos que presentan mucosa gástrica heterotópica existe secreción ácida, lo que favorece la ulceración local y la aparición de complicaciones hemorrágicas. En aquellos que presentan mucosa pancreática la secreción alcalina puede producir esta misma complicación. ${ }^{2}$

Si bien el sangrado es una complicación frecuente del divertículo de Meckel en los niños, en adultos es poco frecuente y no suele ser considerado entre las principales etiologías de hemorragia digestiva baja.

Nuestro objetivo es comentar una presentación atípica del divertículo de Meckel en el adulto, dado por una hemorragia digestiva baja con shock hemorrágico.

\section{CASO CLÍNICO}

Se presenta el caso de un paciente de 34 años, sin antecedentes, que consulta en emergencia por dolor abdominal cólico difuso de 72 horas de evolución, que agrega enterorragia, dada por sangre mezclada con la materia fecal 


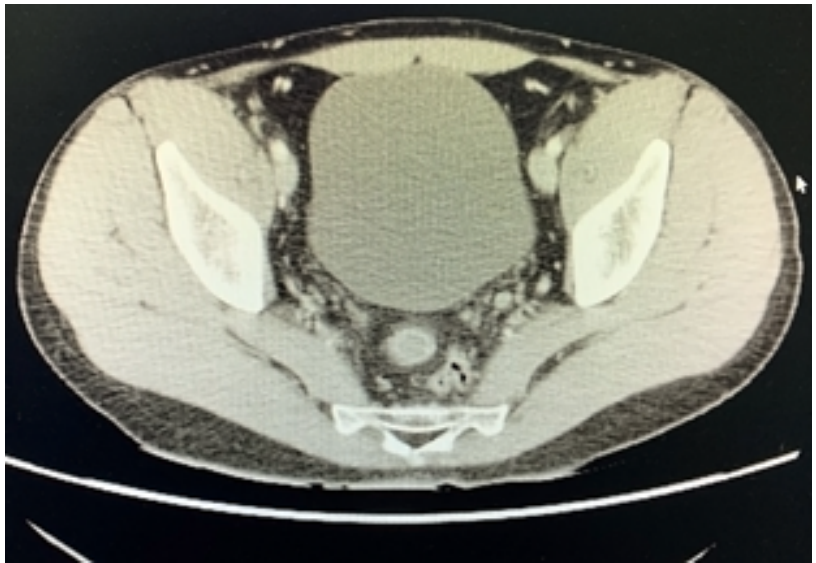

Figura 1: TC de abdomen que evidencia asa delgada de paredes engrosadas y alteración de la grasa adyacente a nivel de pelvis (flecha).

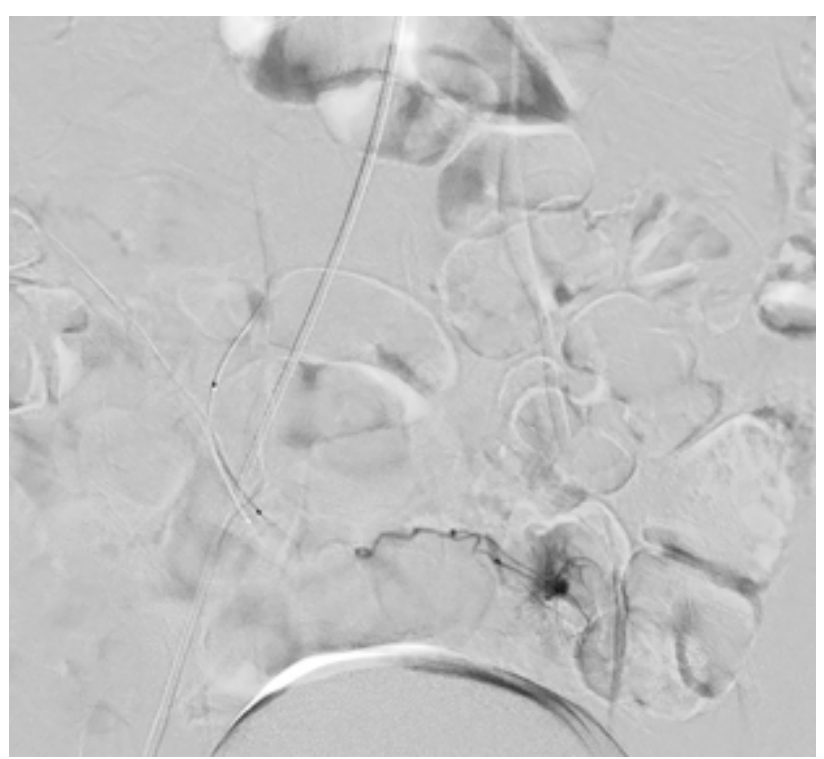

Figura 2: Arteriografía que evidencia extravasación de contraste (flecha).

con coágulos frescos. Se asocia además un síndrome funcional anémico.

Al examen al ingreso presenta hipocoloración mucosa, taquicardia de $100 \mathrm{cpm}$, normotenso y dolor abdominal en hemiabdomen inferior, sin defensa.

De la paraclínica humoral se destaca anemia con $\mathrm{Hb}$ 7,9 gr/dl.

Se realiza una Tomografía Computada (TC) que evidencia asa delgada distendida, de paredes engrosadas, topografiada a nivel de hipogastrio, que impresiona corresponder a íleon distal. Asocia mínima alteración de la grasa a nivel pelviano. No se evidencian lesiones en colon. No hay líquido libre ni neumoperitoneo (Figura 1).

Se inicia reposición hidroelectrolítica y con hemoderivados, transfundiéndose dos volúmenes de glóbulos rojos. Posteriormente, ingresa a sala para completar la valoración.

En las primeras horas luego del ingreso, el paciente presenta un nuevo episodio de enterorragia, masiva, insta-

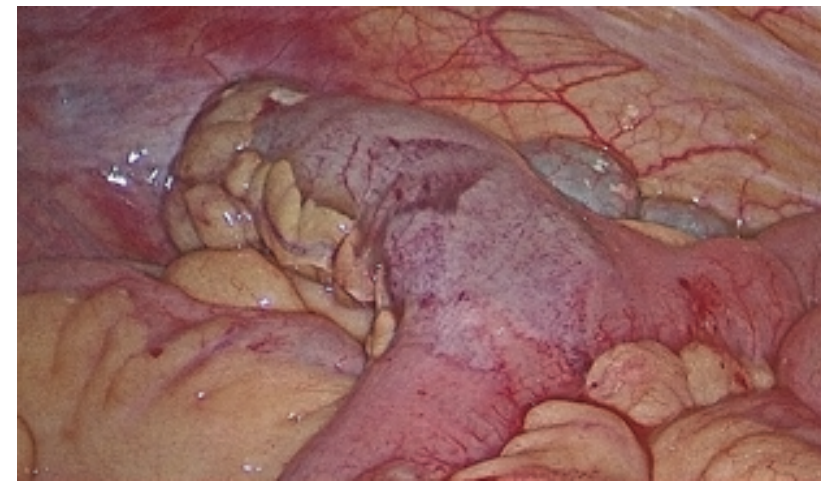

Figura 3: Visión laparoscópica del divertículo de Meckel.

lando un shock hemorrágico que mejora con medidas iniciales de reanimación.

Se decide la solicitud de arteriografía de vasos mesentéricos con eventual angioembolización.

Durante el procedimiento se identifica arteria irregular tortuosa, dependiente de la mesentérica superior, que se localiza a nivel pelviano, inmediatamente por encima de la vejiga, con extravasación espontánea de contraste y pasaje del mismo a un asa delgada (Figura 2).

Se accede a la arteria sangrante a través de microcatéter Echelon 10 sobre una microguía Traxcess 14, y se emboliza con Histoacryl al 30 \%, con oclusión de la misma y cese del sangrado.

El paciente presenta una buena evolución inicial, sin evidencias de resangrado. A las 48 horas instala dolor abdominal a nivel de hipogastrio, con defensa a dicho nivel.

Con planteo de posible isquemia de asa delgada, se decide laparoscopía diagnóstica.

La laparoscopía evidencia escaso exudado fibrinopurulento en pelvis y un divertículo de Meckel a dicho nivel con isquemia parietal que compromete la totalidad del mismo (Figura 3). Se decide su resección hasta su base, por lo que se convierte a una minilaparotomía, dado por una mediana infraumbilical. Se exterioriza el divertículo y se realiza resección en cuña del mismo, conservando espolón mesentérico del asa delgada. Se confecciona una anastomosis término terminal manual con Vicryl 4-0.

El paciente evoluciona favorablemente en el postoperatorio, otorgándole el alta al cuarto día.

\section{DISCUSIÓN}

La hemorragia digestiva baja es un problema diagnóstico y terapéutico en la emergencia. Existe controversia en el manejo inicial de estos pacientes, dependiendo principalmente del estado hemodinámico al ingreso. ${ }^{3}$

En pacientes hemodinámicamente estables el procedimiento diagnóstico de elección es la videocolonoscopía, con previa preparación colónica para mejorar visibilidad y 
reducir riesgos de perforación. ${ }^{3}$ En pacientes inestables, las guías británicas recomiendan la angio TC de inicio, con eventual angioembolización del vaso sangrante, y una endoscopía digestiva baja en diferido para evaluar la lesión. ${ }^{4}$

$\mathrm{La}$ angio TC tiene como ventaja la posibilidad de visualizar sangrados del intestino delgado, por lo que es de elección si se sospecha un sangrado de esta topografía.

Entre un 5-10 \% de las hemorragias digestivas bajas corresponden al intestino delgado. Las causas más frecuentes de estos sangrados varían según la edad del paciente. En pacientes menores a 40 años las causas más frecuentes son la enfermedad inflamatoria intestinal y el divertículo de Meckel.

La angiografía es un método eficaz para topografiar y tratar estos sangrados, con una tasa de éxito que varía entre 40 y $100 \%$ en la literatura. ${ }^{4}$ Algunas de las complicaciones de la embolización angiográfica son el resangrado o la isquemia intestinal, por lo que los pacientes deben ser vigilados en forma estricta en los primeros días post procedimiento. ${ }^{4}$

En casos de divertículo de Meckel con sangrado activo, la arteriografía permite detectar el origen del mismo, al visualizar el vestigio de la arteria onfalomesentérica. La gammagrafía con Tecnecio 99 es utilizada para el diagnóstico en niños, pero tiene una sensibilidad más baja en adultos, además de una limitada disponibilidad, por lo que no es el estudio de primera elección. ${ }^{5}$
En caso de sangrado activo está descrita la angioembolización, con o sin resección posterior del divertículo. ${ }^{5}$ En casos de inestabilidad hemodinámica, la angioembolización puede utilizarse como terapia puente, contemporizando el tratamiento definitivo, permitiendo estabilizar al paciente antes de la cirugía. De esta manera se puede plantear un tratamiento quirúrgico laparoscópico, con las ventajas conocidas del mismo. ${ }^{5}$

El tratamiento resectivo del divertículo es tema de discusión, pudiendo optarse por la resección segmentaria de intestino delgado y anastomosis, resección en cuña o grapado tangencial. ${ }^{1}$

\section{CONCLUSIÓN}

Se presentó el caso de un paciente joven, con una hemorragia digestiva baja masiva, que correspondía a un divertículo de Meckel complicado. El mismo fue tratado en forma inicial mediante angioembolización y requirió posteriormente resección por isquemia local.

Destacamos la importancia de los algoritmos diagnósticos y terapéuticos al enfrentarnos a una hemorragia digestiva baja con repercusión hemodinámica. A su vez, debemos recordar al divertículo de Meckel como una causa frecuente de hemorragia de intestino delgado en el paciente joven.

\section{REFERENCIAS}

1. Hansen CC, Søreide K. Systematic review of epidemiology, presentation, and management of Meckel's diverticulum in the 21st century. Medicine 2018;97:e12154.

2. Uppal K, Tubbs RS, Matusz P, Shaffer K, Loukas M. Meckel's diverticulum: a review. Clin Anat 2011;24:416-22.

3. Strate LL, Gralnek IM. ACG Clinical guideline: Management of patients with acute lower gastrointestinal bleeding. Am J
Gastroenterol 2016;111:459-74. (correction Am J Gastroenterol 2016;111:755).

4. Oakland K, Chadwick G, East JE, et al. Diagnosis and management of acute lower gastrointestinal bleeding: guidelines from the British Society of Gastroenterology. Gut 2019;68:776-89.

5. Liu X, Chan DK, Tan KK. Angioembolisation of a bleeding Meckel's diverticulum. J Gastrointest Surg 2015;19:2286-87.

\section{COMENTARIO}

La presentación como shock hemorrágico de un divertículo de Meckel complicado es una presentación inusual. El manejo inicial corresponde al de una hemorragia digestiva baja de cualquier otra etiología consistiendo en una videocolonoscopía inicial y, en el caso de que esta no arroje ningún sitio de sangrado, puede realizarse un estudio hemodinámico como se realizó en el caso presentado, el cual sirve como método diagnóstico y terapéutico. De acuerdo a la evolución clínica del paciente en este caso, la resolución quirúrgica es mandatoria. Cabe destacar que la sospecha clínica de esta entidad como causal de hemorragia digestiva juega un papel fundamental, debido a lo infrecuente de esta presentación.

Florencia Ventura

Clínica Bazterrica. CABA, Argentina. 